\title{
Near-Infrared Spectroscopy of the Surface of Mars Derived from TIGER Spectro-Imaging Data
}

\author{
P. Martin and P.C. Pinet
}

UPR 234/CNRS, Observatoire Midi-Pyrénées, 31400 Toulouse, France

R. Bacon and A. Rousset

Observatoire de Lyon, 69561 Saint Genis-Laval Cedex, France

\begin{abstract}
High spectral and spatial resolution telescopic observations of the western hemisphere of Mars, using the integral field spectrograph TIGER at 0.8-1.1 $\mu \mathrm{m}$, are described.
\end{abstract}

\section{Observations and data reduction}

In order to assess the spectral variability between bright and dark terrains, we used the opportunity provided by the 1990 perihelic opposition to acquire high spectral resolution $(R=600)$ spectro-imaging data of Mars in the key spectral domain for the understanding of martian surface mineralogy (0.8-1.1 $\mu \mathrm{m})$. A systematic exploration of the Tharsis volcanic dome has been made, including the transition area toward the southern dark terrains in Mare Sirenum and Icaria, by means of the integral field spectrograph TIGER mounted on the $3.6 \mathrm{~m}$ CFH telescope. Spectra were normalized to a spectrally homogeneous region in Tharsis and calibrated with a mean spectrum of this region (produced from the ISM spectrometer coverage). On the basis of an extensive spectro-imaging mapping (about 2000 reflectance spectra with 100 to $300 \mathrm{~km}$ spatial resolution), a linear mixing model is performed, revealing that two extreme bright and dark endmembers account for nearly all the variance in the spectra population at the scale of the analysis. Moreover, to investigate the associations between spectral variations and both morphological and mineralogical features of the surface, several representative mosaic images are produced from this data set.

\section{Discussion}

The interpretation of the bright endmember fraction image indicates a strong correlation with the regional albedo markings. Typical bright regions display fractional abundance values ranging from $70 \%$ to $100 \%$ contribution of the bright endmember, and darkest terrains can be accounted for by areal coverage of at most $50 \%$ of bright region materials. Besides, this mixing model approach allows to detect the more spectroscopically typical zones, particularly within the dark terrains such as Mare Sirenum and Icaria. Different spectral analyses lead to the conclusion of a bimodal distribution of the maximum absorption positions 
in the 0.84-1.0 $\mu \mathrm{m}$ interval, correlated with the global reflectance variations. Indeed, high albedo terrains across Tharsis present a weak short-wavelength absorption band between 0.84 and $0.9 \mu \mathrm{m}$ while a deeper well-defined absorption band is detected between 0.96 and 1 micron in low albedo areas (Figures 1.a and 1.b). The results show a progressive modification of the spectra morphology when crossing the transition zone between dark and bright terrains, supporting the proposed interpretation of the existence of a relative variable contribution of $\mathrm{Fe}^{2+}$ and $\mathrm{Fe}^{3+}$ absorption features in the mineralogical properties of the martian surface (Pinet \& Chevrel 1990). Spatial distributions produced provide the evidence for a hematite-like ferric material domination within the bright areas and for the presence of varied calcic-pyroxenes in ferrous bearing-minerals of the dark terrains.
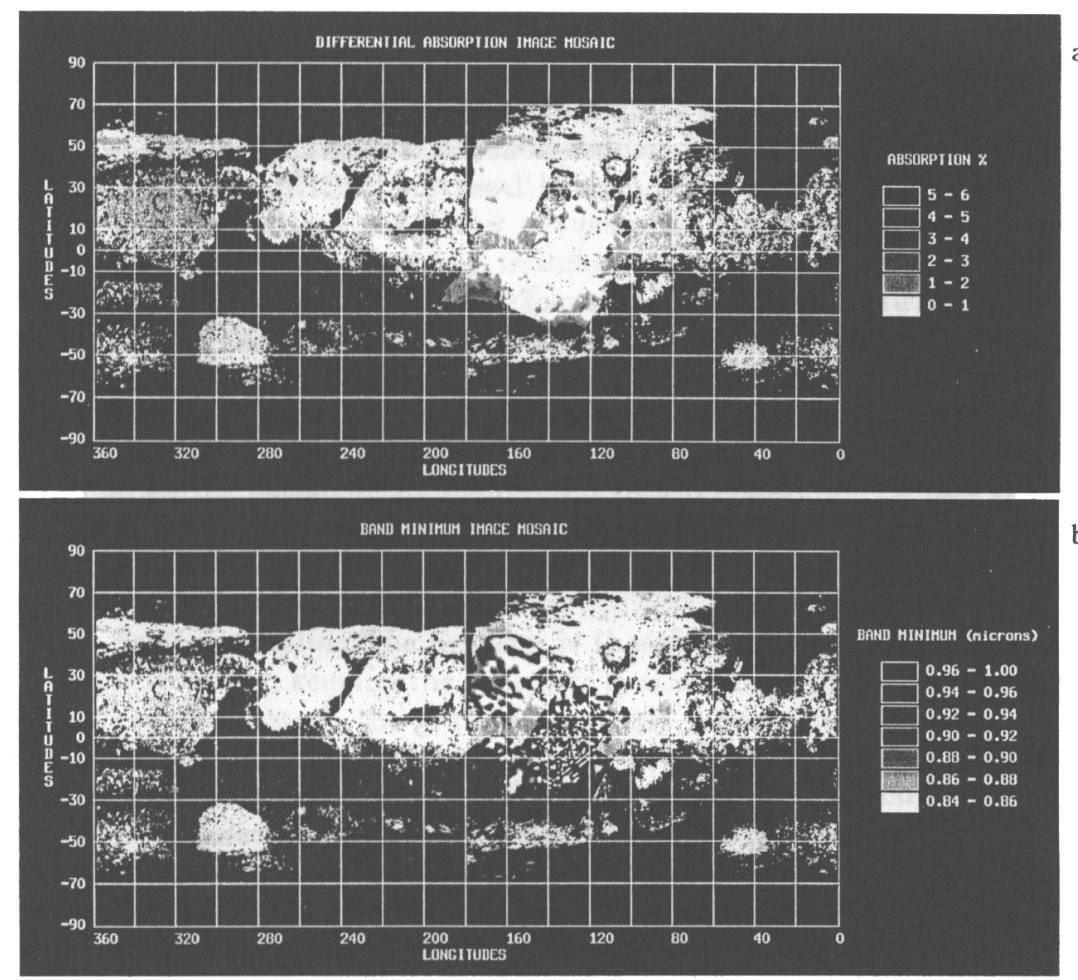

Figure 1. Spatial distributions produced from 2 spectral analyses within the studied region, overlain on a global albedo map of Mars. (a) maximum differential absorption mapping, (b) cartography of the position of this maximum absorption in the domain 0.84-1.0 $\mu \mathrm{m}$.

\section{References}

Pinet, P., \& Chevrel, S. (1990), J. Geophys. Res., vol. 95, no. B9, 14435. 